Korean J. Math. 19 (2011), No. 2, pp. 219-232

\title{
THE PROPERTIES OF ROUGH APPROXIMATIONS
}

\author{
Yong Chan Kim*and Jung Mi Ko
}

\begin{abstract}
We investigated the properties of rough approximations induced by two families of preordered sets and closure systems. We study the relations among the lower and upper rough approximations, closure and interior systems, preordered sets.
\end{abstract}

\section{Introduction}

Rough set theory was introduced by Pawlak [7] to generalize the classical set theory. Rough approximations are defined by a partition of the universe which is corresponding to the equivalence relation about information. J. Järvinen et.al.[3] define rough approximations on preorder relations that are not necessarily equivalence relations. An information consists of $(X, A)$ where $X$ is a set of objects and $A$ is a set of attributes, a map $a: X \rightarrow P\left(A_{a}\right)$ where $A_{a}$ is the value set of the attribute $a$. For $B \subset A$,

$$
\begin{gathered}
(x, y) \in R \Leftrightarrow(\forall a \in B)(a(x)=a(y)) \quad \text { (Pawlaki's sense) } \\
(x, y) \in R \Leftrightarrow(\forall a \in B)(a(x) \subset a(y)) \quad \text { (Järvinen's sense). }
\end{gathered}
$$

It is an important mathematical tool for data analysis and knowledge processing [1-8].

In this paper, we investigated the properties of rough approximations induced by two families of preordered sets and closure systems. We study the relations among the lower and upper rough approximations, closure and interior systems, preordered sets.

Received March 17, 2011. Revised June 16, 2011. Accepted June 17, 2011.

2000 Mathematics Subject Classification: 06A06, 06A15, 06B30, 54F05,68U35.

Key words and phrases: Preordered sets, lower and upper rough approximations, closure and interior systems, product and subspaces of preordered spaces.

*Corresponding author. 
Let $X$ be a set. A relation $e_{X} \subset X \times X$ is called a preorder if it is reflexive and transitive. We can define a preorder $e_{P(X)} \subset P(X) \times$ $P(X)$ as $(A, B) \in e_{P(X)}$ iff $A \subset B$ for $A, B \in P(X)$. If $\left(X, e_{X}\right)$ is a preordered set and we define a function $(x, y) \in e_{X}^{-1}$ iff $(y, x) \in e_{X}$, then $\left(X, e_{X}^{-1}\right)$ is a preordered set.

\section{Preliminaries}

Definition 2.1 [6]. (1) A family $\mathcal{F}=\{A \in P(X)\}$ is called an interior system on $X$ if $\bigcup_{i \in \Gamma} A_{i} \in \mathcal{F}$ for $\left\{A_{i} \mid i \in \Gamma\right\} \subset \mathcal{F}$. Let $\mathcal{F}_{X}$ and $\mathcal{F}_{Y}$ be interior systems on $X$ and $Y$, respectively. A function $f:\left(X, \mathcal{F}_{X}\right) \rightarrow\left(Y, \mathcal{F}_{Y}\right)$ is called an I-map if $f^{-1}(A) \in \mathcal{F}_{X}$ for each $A \in \mathcal{F}_{Y}$.

(2) A family $\mathcal{G}=\{A \in P(X)\}$ is called a closure system on $X$ if $\bigcap_{i \in \Gamma} A_{i} \in \mathcal{G}$ for $\left\{A_{i} \mid i \in \Gamma\right\} \subset \mathcal{G}$. Let $\mathcal{G}_{X}$ and $\mathcal{G}_{Y}$ be closure systems on $X$ and $Y$, respectively. A function $f:\left(X, \mathcal{G}_{X}\right) \rightarrow\left(Y, \mathcal{G}_{Y}\right)$ is called a $C$-map if $f^{-1}(B) \in \mathcal{G}_{X}$ for each $B \in \mathcal{G}_{Y}$. Let $\mathcal{F}_{1}$ (resp. $\mathcal{G}_{1}$ ) and $\mathcal{F}_{2}$ (resp. $\mathcal{G}_{2}$ ) be interior (resp. closure) systems on $X$. $\mathcal{F}_{1}$ (resp. $\mathcal{G}_{1}$ ) is coarser than $\mathcal{F}_{2}$ (resp. $\mathcal{G}_{2}$ ) if $\mathcal{F}_{1} \subset \mathcal{F}_{2}$ (resp. $\mathcal{G}_{1} \subset \mathcal{G}_{2}$ ).

Definition $2.2[4,6]$. Let $\left(X, e_{X}\right)$ be a preodered set. A set $A \in$ $P(X)$ is called an $e_{X}$-upper set if $\left(x \in A \&(x, y) \in e_{X}\right) \rightarrow y \in A$ for $x, y \in X$.

Theorem 2.3 [4-6]. Let $\left(X, e_{X}\right)$ be a preordered set. For $A \in$ $P(X)$, we define operations $\left[e_{X}\right],\left\langle e_{X}\right\rangle$ as follows:

$$
\begin{aligned}
& {\left[e_{X}\right](A)=\left\{x \in X \mid(\forall z \in X)\left((x, z) \in e_{X} \rightarrow z \in A\right)\right\},} \\
& \left\langle e_{X}\right\rangle(A)=\left\{x \in X \mid(\exists z \in X)\left((x, z) \in e_{X} \& z \in A\right)\right\} .
\end{aligned}
$$

Then the following properties hold.

(1)If $\left(e_{X}\right)_{x}=\left\{z \in X \mid(x, z) \in e_{X}\right\}$ and $\left(e_{X}\right)_{x}^{-1}=\{z \in X \mid(z, x) \in$ $\left.e_{X}\right\}$, then $\left(e_{X}\right)_{x}$ and $\left(\left(e_{X}\right)_{x}^{-1}\right)^{c}$ are $e_{X}$-upper sets.

(2) $A$ is an $e_{X}$-upper set iff $\left[e_{X}\right](A)=A$ iff $\left[e_{X}^{-1}\right]\left(A^{c}\right)=A^{c}$ iff $\left\langle e_{X}^{-1}\right\rangle(A)=A$.

(3) If $A_{i}$ is an $e_{X}$-upper set for all $i \in \Gamma$, then $\bigcup_{i \in \Gamma} A_{i}$ and $\bigcap_{i \in \Gamma} A_{i}$ are $e_{X}$-upper sets.

(4) $\left[e_{X}\right](A)=\bigcup_{i}\left\{A_{i} \mid A_{i} \subset A, A_{i}: e_{X}-\right.$ upper set $\}$.

(5) $\left\langle e_{X}\right\rangle(A)=\bigcap_{i}\left\{A_{i} \mid A \subset A_{i}, A_{i}: e_{X}^{-1}-\right.$ upper set $\}$. 
Definition $2.4[3,6]$. In above theorem, $\left[e_{X}\right](A)$ and $\left\langle e_{X}\right\rangle(A)$ are called rough lower approximation and rough upper approximation, respectively, for $A \in P(X)$ on a preodered set.

If $e_{X}$ is an equivalence relation, $\left[e_{X}\right](A)$ and $\left\langle e_{X}\right\rangle(A)$ are rough lower approximation and rough upper approximation for $A \in P(X)$ in a Pawlak's sense [7]. Let $\left(X, e_{X}\right)$ and $\left(Y, e_{Y}\right)$ be preordered sets. A function $f:\left(X, e_{X}\right) \rightarrow\left(Y, e_{Y}\right)$ is called an order preserving map if $(f(x), f(y)) \in e_{Y}$ for all $(x, y) \in e_{X}$.

Theorem 2.5 [6]. Let $\mathcal{F}=\left\{B_{i} \in P(X) \mid i \in \Gamma\right\}$ be an interior system and $\mathcal{F}^{*}=\left\{A_{i} \in P(X) \mid A_{i}^{c} \in \mathcal{F}\right\}$.

Then; (1) $\mathcal{F}^{*}$ is a closure system.

(2) There exists a preorder $e_{\mathcal{F}}$ on $X$ such that $\mathcal{F} \subset \mathcal{F}_{\left[e_{\mathcal{F}}\right]}$ with

$$
(x, y) \in e_{\mathcal{F}} \quad \text { iff }\left(\forall B_{i} \in \mathcal{F}\right)\left(x \in B_{i} \rightarrow y \in B_{i}\right)
$$

(3) There exists a preorder $e_{\mathcal{F}^{*}}$ on $X$ such that $\mathcal{F}^{*} \subset \mathcal{F}_{\left\langle e_{\left.\mathcal{F}^{*}\right\rangle}^{*}\right.}^{*}$ with

$$
(x, y) \in e_{\mathcal{F}^{*}} \quad \text { iff }\left(\forall A_{i} \in \mathcal{F}^{*}\right)\left(y \in A_{i} \rightarrow x \in A_{i}\right) .
$$

Theorem $2.6[6]$. Let $\left(X, e_{X}\right)$ be a preordered set. Define $\mathcal{F}_{\left[e_{X}\right]}, \mathcal{G}_{\left\langle e_{X}\right\rangle}$ as follows:

$$
\begin{gathered}
\mathcal{F}_{\left[e_{X}\right]}=\left\{A \in P(X) \mid\left[e_{X}\right](A)=A\right\} \\
\mathcal{G}_{\left\langle e_{X}\right\rangle}=\left\{A \in P(X) \mid\left\langle e_{X}\right\rangle(A)=A\right\}
\end{gathered}
$$

Then; (1) $\left[e_{X}\right](A) \subset A$ and $\left[e_{X}\right](A)=\left[e_{X}\right]\left(\left[e_{X}\right](A)\right)$, for each $A \in$ $P(X)$ with $x \in\left[e_{X}\right]\left(\{y\}^{c}\right)$ iff $(x, y) \notin e_{X}$.

(2) $A \subset\left\langle e_{X}\right\rangle(A)$ and $\left\langle e_{X}\right\rangle(A)=\left\langle e_{X}\right\rangle\left(\left\langle e_{X}\right\rangle(A)\right)$, for each $A \in P(X)$ with $x \in\left\langle e_{X}\right\rangle(\{y\})$ iff $(x, y) \in e_{X}$.

(3) $\mathcal{F}_{\left[e_{X}\right]}$ is an interior and closure system with $I_{\mathcal{F}_{\left[e_{X}\right]}}=\left[e_{X}\right]$ where $I_{\mathcal{F}_{\left[e_{X}\right]}}(A)=\bigcup\left\{B \mid B \subset A, B \in \mathcal{F}_{\left[e_{X}\right]}\right\}$.

(4) $\mathcal{G}_{\left\langle e_{X}\right\rangle}$ is an interior and closure system with $C_{\mathcal{G}_{\left\langle e_{X}\right\rangle}}=\left\langle e_{X}\right\rangle$ where $C_{\mathcal{G}_{\left\langle e_{X}\right\rangle}}(A)=\bigcap\left\{B \mid A \subset B, B \in \mathcal{G}_{\left\langle e_{X}\right\rangle}\right\}$.

(5) $e_{\mathcal{F}_{\left[e_{X}\right]}}=e_{X}$ and $e_{\mathcal{G}_{\left\langle e_{X}\right\rangle}}=e_{X}$. 


\section{The properties of rough approximations}

Theorem 3.1. Let $X$ be a set and $\left\{\left(X_{i}, \mathcal{G}_{i}\right)\right\}_{i \in \Gamma}$ a family of closure systems. Let $f_{i}: X \rightarrow X_{i}$ be a function. Define

$$
\mathcal{G}=\left\{\bigcap_{i \in \Gamma} f_{i}^{-1}\left(B_{i}\right) \mid B_{i} \in \mathcal{G}_{i}\right\}
$$

Then $(1) \mathcal{G}$ is the coarsest closure system on $X$ for which each $f_{i}$ is a C-map.

(2) $\mathcal{G}^{*}=\left\{\bigcup_{i \in \Gamma} f_{i}^{-1}\left(A_{i}\right) \mid A_{i} \in \mathcal{G}_{i}^{*}\right\}$. is the coarsest interior system on $X$ for which each $f_{i}$ is an I-map.

(3) A function $f:\left(Y, \mathcal{G}^{\prime}\right) \rightarrow(X, \mathcal{G})$ is a $C$-map iff $f_{i} \circ f:\left(Y, \mathcal{G}^{\prime}\right) \rightarrow$ $\left(X_{i}, \mathcal{G}_{i}\right)$ is a $C$-map, for each $i \in \Gamma$.

(4) A function $f:\left(Y, \mathcal{F}^{\prime}\right) \rightarrow\left(X, \mathcal{G}^{*}\right)$ is an I-map iff $f_{i} \circ f:(Y, \mathcal{F}) \rightarrow$ $\left(X_{i}, \mathcal{G}_{i}^{*}\right)$ is an I-map, for each $i \in \Gamma$.

(5) $(x, y) \in e_{\mathcal{G}}$ iff $(\forall i \in \Gamma)\left(\left(f_{i}(x), f_{i}(y)\right) \in e_{\mathcal{G}_{X_{i}}}\right)$.

(6) $(x, y) \in e_{\mathcal{G}^{*}}$ iff $(\forall i \in \Gamma)\left(\left(f_{i}(x), f_{i}(y)\right) \in e_{\mathcal{G}_{X_{i}}^{*}}\right)$. In particular, $e_{\mathcal{G}}{ }=e_{\mathcal{G}}$.

(7) $\mathcal{G} \subset \mathcal{G}_{\left\langle e_{\mathcal{G}}\right\rangle}=\left\{\bigcap_{i \in \Gamma} f_{i}^{-1}\left(B_{i}\right), \bigcup_{i \in \Gamma} f_{i}^{-1}\left(B_{i}\right) \mid B_{i} \in \mathcal{G}_{i}\right\}$.

(8) $\mathcal{G}^{*} \subset \mathcal{F}_{\left[e_{\mathcal{G}^{*}}\right]}=\left\{\bigcap_{i \in \Gamma} f_{i}^{-1}\left(A_{i}\right), \bigcup_{i \in \Gamma} f_{i}^{-1}\left(A_{i}\right) \mid A_{i} \in \mathcal{G}_{i}^{*}\right\}=\{A \mid$ $\left.A^{c} \in \mathcal{G}_{\left\langle e_{\mathcal{G}}\right\rangle}\right\}$.

Proof. (1) For each $k \in K$ with index set $K, A_{k}=\bigcap_{i \in \Gamma} f_{i}^{-1}\left(B_{i k}\right) \in$ $\mathcal{G}$, then $\bigcap_{k \in K} A_{k}=\bigcap_{i \in \Gamma} f_{i}^{-1}\left(\bigcap_{k \in K} B_{i k}\right) \in \mathcal{G}$. Hence $\mathcal{G}$ is a closure system on $X$. For $B_{i} \in \mathcal{G}_{i}$, since $X_{j} \in \mathcal{G}_{j}$ because it is the intersection of empty family, then $f_{i}^{-1}\left(B_{i}\right)=f_{i}^{-1}\left(B_{i}\right) \cap\left(\bigcap_{j \in \Gamma-\{i\}} f_{j}^{-1}\left(X_{j}\right)\right) \in \mathcal{G}$. Hence $f_{i}$ is a C-map. Let $f_{i}:\left(X, \mathcal{G}^{\prime}\right) \rightarrow\left(X_{i}, \mathcal{G}_{i}\right)$ be a C-map. For $B=\bigcap_{i \in \Gamma} f_{i}^{-1}\left(B_{i}\right) \in \mathcal{G}$ with $B_{i} \in \mathcal{G}_{i}$, then $f_{i}^{-1}\left(B_{i}\right) \in \mathcal{G}^{\prime}$. Since $\mathcal{G}^{\prime}$ is a closure system on $X, B \in \mathcal{G}^{\prime}$, that is, $\mathcal{G} \subset \mathcal{G}^{\prime}$. Hence $\mathcal{G}$ is the coarsest closure system on $X$ for which each $f_{i}$ is a C-map.

$(3)(\Rightarrow)$ It is trivial because the composition of C-maps is a C-map.

$(\Leftarrow)$ For $B=\bigcap_{i \in \Gamma} f_{i}^{-1}\left(B_{i}\right) \in \mathcal{G}$ with $B_{i} \in \mathcal{G}_{i}$, then $f^{-1}\left(f_{i}^{-1}\left(B_{i}\right)\right) \in$ $\mathcal{G}^{\prime}$. It implies $f^{-1}(B)=\bigcap_{i \in \Gamma} f^{-1}\left(f_{i}^{-1}\left(B_{i}\right)\right) \in \mathcal{G}^{\prime}$. Thus, $f$ is a C-map.

(2) and (4) are similarly proved as in (1) and (3), respectively.

(5) Suppose there exist $x, y \in X$ such that $(x, y) \in e_{\mathcal{G}}$, but $\forall(\forall i \in$ $\Gamma)\left(\left(f_{i}(x), f_{i}(y)\right) \in e_{\mathcal{G}_{X_{i}}}\right)$;i.e.

$$
\forall(\forall i \in \Gamma)\left(\forall B_{i} \in \mathcal{G}_{i}\right)\left(f_{i}(y) \in B_{i} \rightarrow f_{i}(x) \in B_{i}\right) .
$$


Then there exists $i_{0} \in \Gamma$ such that $(x, y) \in e_{\mathcal{G}}$,

$$
\forall\left(\forall B \in \mathcal{G}_{i_{0}}\right)\left(f_{i_{0}}(y) \in B \rightarrow f_{i_{0}}(x) \in B\right) .
$$

Then there exists $B_{i_{0}} \in \mathcal{G}_{i_{0}}$ such that $(x, y) \in e_{\mathcal{G}}$,

$$
f_{i_{0}}(y) \in B_{i_{0}} \rightarrow f_{i_{0}}(x) \notin B_{i_{0}} .
$$

Put $B=f_{i_{0}}^{-1}\left(B_{i_{0}}\right) \cap\left(\bigcap_{i \neq i_{0}} f_{i}^{-1}\left(X_{i}\right)\right)$. Then

$$
\vdash(x, y) \in e_{\mathcal{G}} \rightarrow(y \in B \rightarrow x \in B) .
$$

Since $\vdash(x, y) \in e_{\mathcal{G}}$, by Modus Ponens, $\vdash\left(f_{i_{0}}(y) \in B_{i_{0}} \rightarrow f_{i_{0}}(x) \in B_{i_{0}}\right)$. It is a contradiction. Hence, if $(x, y) \in e_{\mathcal{G}}$, then $(\forall i \in \Gamma)\left(\left(f_{i}(x), f_{i}(y)\right) \in\right.$ $\left.e_{\mathcal{G}_{X_{i}}}\right)$.

Suppose there exist $x, y \in X$ such that

$$
(x, y) \notin e_{\mathcal{G}}, \vdash(\forall i \in \Gamma)\left(\left(f_{i}(x), f_{i}(y)\right) \in e_{\mathcal{G}_{X_{i}}}\right) .
$$

By the definition of $e_{\mathcal{G}}$, there exists $B=\bigcap_{i \in \Gamma} f_{i}^{-1}\left(B_{i}\right) \in \mathcal{G}$ such that

$$
\begin{gathered}
\forall \forall\left(y \in \bigcap_{i \in \Gamma} f_{i}^{-1}\left(B_{i}\right) \rightarrow x \in \bigcap_{i \in \Gamma} f_{i}^{-1}\left(B_{i}\right)\right) \\
\vdash(\forall i \in \Gamma)\left(\left(f_{i}(x), f_{i}(y)\right) \in e_{\mathcal{G}_{X_{i}}}\right) .
\end{gathered}
$$

Since

$$
\begin{gathered}
\vdash \bigcap_{i \in \Gamma}\left(y \in f_{i}^{-1}\left(B_{i}\right) \rightarrow x \in f_{i}^{-1}\left(B_{i}\right)\right) \rightarrow \\
\left(y \in \bigcap_{i \in \Gamma} f_{i}^{-1}\left(B_{i}\right) \rightarrow x \in \bigcap_{i \in \Gamma} f_{i}^{-1}\left(B_{i}\right)\right)
\end{gathered}
$$

and $\vdash(\forall i \in \Gamma)\left(\left(f_{i}(x), f_{i}(y)\right) \in e_{\mathcal{G}_{X_{i}}}\right)$, by Modus Ponens,

$$
\vdash\left(y \in \bigcap_{i \in \Gamma} f_{i}^{-1}\left(B_{i}\right) \rightarrow x \in \bigcap_{i \in \Gamma} f_{i}^{-1}\left(B_{i}\right)\right)
$$


It is a contradiction. Hence, if $(\forall i \in \Gamma)\left(\left(f_{i}(x), f_{i}(y)\right) \in e_{\mathcal{G}_{X_{i}}}\right)$, then $(x, y) \in e_{\mathcal{G}}$.

(6) Suppose there exist $x, y \in X$ such that

$$
(x, y) \in e_{\mathcal{G}^{*}}, \quad \forall(\forall i \in \Gamma)\left(\left(f_{i}(x), f_{i}(y)\right) \in e_{\mathcal{G}_{X_{i}}^{*}} .\right.
$$

Then there exists $i_{0} \in \Gamma$ such that

$$
(x, y) \in e_{\mathcal{G}^{*}}, \quad\left(f_{i_{0}}(x), f_{i_{0}}(y)\right) \notin e_{\mathcal{G}_{X_{i_{0}}}^{*}} .
$$

Then there exists $A_{i_{0}} \in \mathcal{G}_{i_{0}}^{*}$ such that

$$
(x, y) \in e_{\mathcal{G}^{*}}, \forall\left(f_{i_{0}}(x) \in A_{i_{0}} \rightarrow f_{i_{0}}(y) \in A_{i_{0}}\right) .
$$

Put $A=f_{i_{0}}^{-1}\left(A_{i_{0}}\right) \cup\left(\bigcup_{i \neq i_{0}} f_{i}^{-1}(\emptyset)\right)$. Then $\vdash\left((x, y) \in e_{\mathcal{G}^{*}} \rightarrow\left(f_{i_{0}}(x) \in\right.\right.$ $\left.\left.A_{i_{0}} \rightarrow f_{i_{0}}(y) \in A_{i_{0}}\right)\right)$. By Modus Ponens, $\vdash\left(f_{i_{0}}(x) \in A_{i_{0}} \rightarrow f_{i_{0}}(y) \in\right.$ $\left.\left.A_{i_{0}}\right)\right)$. It is a contradiction.

Hence, if $(x, y) \in e_{\mathcal{G}^{*}}$, then $(\forall i \in \Gamma)\left(\left(f_{i}(x), f_{i}(y)\right) \in e_{\mathcal{G}_{X_{i}}^{*}}\right)$.

Suppose there exist $x, y \in X$ such that

$$
(x, y) \notin e_{\mathcal{G}^{*}}, \vdash(\forall i \in \Gamma)\left(\left(f_{i}(x), f_{i}(y)\right) \in e_{\mathcal{G}_{X_{i}}^{*}}\right) .
$$

Then there exists $A=\bigcup_{i \in \Gamma} f_{i}^{-1}\left(A_{i}\right) \in \mathcal{G}^{*}$ such that

$$
\begin{gathered}
\forall\left(x \in \bigcup_{i \in \Gamma} f_{i}^{-1}\left(A_{i}\right) \rightarrow y \in \bigcup_{i \in \Gamma} f_{i}^{-1}\left(A_{i}\right)\right) \\
\vdash(\forall i \in \Gamma)\left(\left(f_{i}(x), f_{i}(y)\right) \in e_{\mathcal{G}_{X_{i}}^{*}}\right) .
\end{gathered}
$$

Since

$$
\begin{aligned}
& \vdash \bigcap_{i \in \Gamma}\left(x \in f_{i}^{-1}\left(A_{i}\right) \rightarrow y \in f_{i}^{-1}\left(A_{i}\right)\right) \rightarrow \\
& \left(x \in \bigcup_{i \in \Gamma} f_{i}^{-1}\left(A_{i}\right) \rightarrow y \in \bigcup_{i \in \Gamma} f_{i}^{-1}\left(A_{i}\right)\right)
\end{aligned}
$$

By Modus Ponens,

$$
\vdash\left(x \in \bigcup_{i \in \Gamma} f_{i}^{-1}\left(A_{i}\right) \rightarrow y \in \bigcup_{i \in \Gamma} f_{i}^{-1}\left(A_{i}\right)\right) .
$$


It is a contradiction. Hence, if $(\forall i \in \Gamma)\left(\left(f_{i}(x), f_{i}(y)\right) \in e_{\mathcal{G}_{X_{i}}^{*}}\right)$, then $(x, y) \in e_{\mathcal{G}^{*}}$.

We have $(x, y) \in e_{\mathcal{G}^{*}}$ iff $\vdash\left(\forall B \in \mathcal{G}^{*}\right)(x \in B \rightarrow y \in B)$ iff $\vdash\left(\forall B^{*} \in\right.$ $\mathcal{G})\left(y \in B^{*} \rightarrow x \in B^{*}\right)$ iff $(x, y) \in e_{\mathcal{G}}$.

(7) Let $B \in \mathcal{G}$. Then $B \subset\left\{x \in X \mid(\exists y \in X)\left((x, y) \in e_{\mathcal{G}} \& y \in B\right)\right\}$ because

$$
\vdash\left((x, x) \in e_{\mathcal{G}} \& x \in B\right) \rightarrow(\exists y \in X)\left((x, y) \in e_{\mathcal{G}} \& y \in B\right)
$$

Since $B \in \mathcal{G}$, then

$$
\begin{gathered}
\vdash\left((x, y) \in e_{\mathcal{G}} \& y \in B\right) \rightarrow(y \in B \rightarrow x \in B) \& y \in B \\
\vdash(y \in B \rightarrow x \in B) \& y \in B \rightarrow x \in B
\end{gathered}
$$

By Modus Ponens, $\vdash\left((x, y) \in e_{\mathcal{G}} \& y \in B\right) \rightarrow x \in B$. Thus, $\{x \in$ $\left.X \mid(\exists y \in X)\left((x, y) \in e_{\mathcal{G}} \& y \in B\right)\right\} \subset B$. Hence $B=\left\langle e_{\mathcal{G}}\right\rangle(B)$. Thus, $B \in \mathcal{G}_{\left\langle e_{\mathcal{G}}\right\rangle}$. So, $\mathcal{G} \subset \mathcal{G}_{\left\langle e_{\mathcal{G}}\right\rangle}$.

For $B=\bigcup_{i \in \Gamma} f_{i}^{-1}\left(B_{i}\right)$ with $B_{i} \in \mathcal{G}_{i},\left(y \in \bigcup_{i \in \Gamma} f_{i}^{-1}\left(B_{i}\right)\right) \&(x, y) \in$ $e_{\mathcal{G}}$ implies $(\exists i \in \Gamma)\left(f_{i}(y) \in B_{i} \&\left(f_{i}(x), f_{i}(y)\right) \in e_{\mathcal{G}_{i}}\right.$ implies $(\exists i \in$ $\Gamma)\left(f_{i}(y) \in B_{i} \&\left(f_{i}(y) \in B_{i} \rightarrow f_{i}(x) \in B_{i}\right)\right.$ implies $x \in \bigcup_{i \in \Gamma} f_{i}^{-1}\left(B_{i}\right)$. Thus $B$ is an $e_{X}^{-1}$-upper set. By Theorem 2.3(2), $B=\left\langle e_{\mathcal{G}}\right\rangle(B)$. Hence $\bigcup_{i \in \Gamma} f_{i}^{-1}\left(B_{i}\right) \in \mathcal{G}_{\left\langle e_{\mathcal{G}}\right\rangle}$.

(8) Let $A \in \mathcal{G}^{*} . \vdash(\forall y \in X)\left(\left((x, y) \in e_{\mathcal{G}^{*}} \rightarrow y \in A\right) \rightarrow x \in A\right)$. Conversely, since $\vdash(\forall y \in X)((x \in A) \&(x \in A \rightarrow y \in A) \rightarrow y \in A)$ and $\vdash(\forall x, y \in X)\left((x, y) \in e_{\mathcal{G}^{*}} \rightarrow(x \in A \rightarrow y \in A)\right.$, we have $\vdash(\forall y \in$ $X)\left(x \in A \&(x, y) \in e_{\mathcal{G}^{*}} \rightarrow y \in A\right.$ iff $\vdash(x \in A) \rightarrow\left((x, y) \in e_{\mathcal{G}^{*}} \rightarrow y \in\right.$ $A)$. Hence $\left[e_{\mathcal{G}^{*}}\right](A)=A$. Thus, $A \in \mathcal{F}_{e_{\mathcal{G}^{*}}}$. So, $\mathcal{G}^{*} \subset \mathcal{F}_{\left[e_{\mathcal{G}^{*}}\right]}$.

For $A=\bigcap_{i \in \Gamma} f_{i}^{-1}\left(A_{i}\right)$ with $A_{i} \in \mathcal{G}_{i}^{*},\left(x \in \bigcup_{i \in \Gamma} f_{i}^{-1}\left(A_{i}\right)\right) \&(x, y) \in$ $e_{\mathcal{G}} *$ implies $(\exists i \in \Gamma)\left(f_{i}(x) \in A_{i} \&\left(f_{i}(x), f_{i}(y)\right) \in e_{\mathcal{G}_{i}^{*}}\right.$ implies $(\exists i \in$ $\Gamma)\left(f_{i}(x) \in A_{i} \&\left(f_{i}(x) \in A_{i} \rightarrow f_{i}(y) \in A_{i}\right)\right.$ implies $y \in \bigcup_{i \in \Gamma} f_{i}^{-1}\left(A_{i}\right)$. Thus $A$ is $e_{X^{-}}$-upper set. By Theorem 2.3(2), $A=\left[e_{\mathcal{G}^{*}}\right](A)$. Hence $A=\bigcap_{i \in \Gamma} f_{i}^{-1}\left(A_{i}\right) \in \mathcal{F}_{\left[e_{\mathcal{G}^{*}}\right]}$.

Example 3.2. Let $X=\{a, b, c, d\}$ be a set, $\mathcal{G}_{1}=\{\{a, b\}, X\}$ and $\mathcal{G}_{2}=\{\{b, c\}, X\}$. Then $\mathcal{G}=\{\{a, b\},\{b, c\},\{b\}, X\}$ be the coarsest 
closure system which is finer than $\mathcal{G}_{i}$ for $i=1,2$. We obtain

$$
\begin{aligned}
e_{\mathcal{G}_{1}}= & \{(a, a)(a, b),(a, c),(a, d),(b, a)(b, b),(b, c),(b, d), \\
& (c, c),(c, d),(d, c),(d, d)\}, \\
e_{\mathcal{G}_{2}}= & \{(a, a)(a, d),(b, a)(b, b),(b, c),(b, d), \\
& (c, a),(c, b)(c, c),(c, d),(d, a),(d, d)\}, \\
e_{\mathcal{G}}= & e_{\mathcal{G}_{1}} \cap e_{\mathcal{G}_{1}}=\{(a, a)(a, d),(b, a)(b, b),(b, c),(b, d), \\
& (c, c),(c, d),(d, d)\}, \\
\mathcal{G}_{1}^{*}= & \{\emptyset,\{c, d\}\}, \quad \mathcal{G}_{2}^{*}=\{\emptyset,\{a, d\}\} .
\end{aligned}
$$

Then $\mathcal{G}^{*}=\{\emptyset,\{c, d\},\{a, d\},\{d\}\}$ is the coarsest interior system which is finer than $\mathcal{G}_{i}^{*}$ for $i=1,2$. In particular, $e_{\mathcal{G}_{1}}=e_{\mathcal{G}_{1}^{*}}, e_{\mathcal{G}_{2}}=e_{\mathcal{G}_{2}^{*}}, e_{\mathcal{G}}=$ $e_{\mathcal{G} *}$ and

$$
\begin{aligned}
\mathcal{G}_{\left\langle e_{\mathcal{G}}\right\rangle} & =\{\emptyset,\{a, b\},\{b, c\},\{b\},\{a, b, c\}, X\} \\
\mathcal{F}_{\left[e_{\mathcal{G}^{*}}\right]} & =\{\emptyset,\{c, d\},\{a, d\},\{a, c, d\},\{d\}, X\} .
\end{aligned}
$$

Theorem 3.3. Let $X$ be a set and $\left\{\left(X_{i}, e_{X_{i}}\right)\right\}_{i \in \Gamma}$ a family of preorder sets. Let $f_{i}: X \rightarrow X_{i}$ be a function for each $i \in \Gamma$. We define the relation $e_{X} \subset X \times X$ by

$$
(x, y) \in e_{X} \text { iff }(\forall i \in \Gamma)\left(\left(f_{i}(x), f_{i}(y)\right) \in e_{X_{i}}\right) .
$$

Then we have the following statements:

(1) $e_{X}$ is the coarsest preorder on $X$ for which each $f_{i}$ is an order preserving map.

(2) A function $f:\left(Y, e_{X}^{\prime}\right) \rightarrow\left(X, e_{X}\right)$ is an order preserving map iff $f_{i} \circ f:\left(Y, e_{X}^{\prime}\right) \rightarrow\left(X_{i}, e_{X_{i}}\right)$ is an order preserving map, for each $i \in \Gamma$.

(3) $e_{X}=e_{\left.\mathcal{G}_{\left\langle e_{X}\right\rangle}\right\rangle}=e_{\mathcal{G}}$ where $\mathcal{G}=\left\{\bigcap_{i \in \Gamma} f_{i}^{-1}\left(B_{i}\right) \mid B_{i} \in \mathcal{G}_{\left\langle e_{X_{i}}\right\rangle}\right\}$.

(4) $\left\langle e_{X}\right\rangle(A) \subset \bigcap_{i \in \Gamma} f_{i}^{-1}\left(\left\langle e_{X_{i}}\right\rangle\left(f_{i}(A)\right)\right)$. If $\Gamma=\{i\}$, the equality holds.

(5) $\left[e_{X}\right](A) \supset \bigcup_{i \in \Gamma} f_{i}^{-1}\left(\left[e_{X_{i}}\right]\left(\left(f_{i}\left(A^{c}\right)^{c}\right)\right)\right)$. If $\Gamma=\{i\}$, the equality holds.

(6) $\bigcap_{i \in \Gamma} f_{i}^{-1}\left(\mathcal{F}_{\left[e_{X_{i}}\right]}\right) \subset \mathcal{F}_{\left[e_{X}\right]}$. If $\Gamma=\{i\}$, the equality holds.

(7) $\bigcup_{i \in \Gamma} f_{i}^{-1}\left(\mathcal{G}_{\left\langle e_{X_{i}}\right\rangle}\right) \subset \mathcal{G}_{\left\langle e_{X}\right\rangle}$. If $\Gamma=\{i\}$, the equality holds. 
Proof. (1) We easily show that $e_{X}$ is the coarsest preorder on $X$ for which each $f_{i}$ is an order preserving map.

$(2)(\Rightarrow)$ It is trivial because the composition of order preserving maps is an order preserving map.

$(\Leftarrow)$ Let $f_{i} \circ f:\left(Y, e_{Y}^{\prime}\right) \rightarrow\left(X_{i}, e_{X_{i}}\right)$ be an order preserving map for each $i \in \Gamma$. For each $x, y \in Y$,

$$
\begin{aligned}
& \vdash(x, y) \in e_{Y}^{\prime} \rightarrow(\forall i \in \Gamma)\left(\left(f_{i}(f(x)), f_{i}(f(y))\right) \in e_{X_{i}}\right) \\
& \text { iff } \quad \vdash(x, y) \in e_{Y}^{\prime} \rightarrow(f(x), f(y)) \in e_{X} .
\end{aligned}
$$

Hence $f:\left(Y, e_{Y}^{\prime}\right) \rightarrow\left(X, e_{X}\right)$ is an order preserving map.

(3) First, we show that $\left(\forall i \in \Gamma, B_{i} \in \mathcal{G}_{\left\langle e_{X_{i}}\right\rangle}\right)\left(y \in \bigcap_{i \in \Gamma} f_{i}^{-1}\left(B_{i}\right) \rightarrow\right.$ $\left.x \in \bigcap_{i \in \Gamma} f_{i}^{-1}\left(B_{i}\right)\right)$ iff $(\forall i \in \Gamma)\left(B_{i} \in \mathcal{G}_{\left\langle e_{X_{i}}\right\rangle}\right)\left(y \in f_{i}^{-1}\left(B_{i}\right) \rightarrow x \in\right.$ $\left.f_{i}^{-1}\left(B_{i}\right)\right)$. Since $f_{i}^{-1}\left(B_{i}\right)=f_{i}^{-1}\left(B_{i}\right) \cap\left(\bigcap_{j \in \Gamma-\{i\}} f_{j}^{-1}\left(X_{j}\right)\right), \quad(\forall i \in$ $\left.\Gamma, B_{i} \in \mathcal{G}_{\left\langle e_{X_{i}}\right\rangle}\right)\left(y \in \bigcap_{i \in \Gamma} f_{i}^{-1}\left(B_{i}\right) \rightarrow x \in \bigcap_{i \in \Gamma} f_{i}^{-1}\left(B_{i}\right)\right)$ implies $(\forall i \in$ $\Gamma)\left(B_{i} \in \mathcal{G}_{\left\langle e_{X_{i}}\right\rangle}\right)\left(y \in f_{i}^{-1}\left(B_{i}\right) \rightarrow x \in f_{i}^{-1}\left(B_{i}\right)\right)$. Conversely, it follows from

$$
\begin{gathered}
\vdash \bigcap_{i \in \Gamma}\left(y \in f_{i}^{-1}\left(B_{i}\right) \rightarrow x \in f_{i}^{-1}\left(B_{i}\right)\right) \\
\rightarrow\left(y \in \bigcap_{i \in \Gamma} f_{i}^{-1}\left(B_{i}\right) \rightarrow x \in \bigcap_{i \in \Gamma} f_{i}^{-1}\left(B_{i}\right)\right)
\end{gathered}
$$

Thus,

$$
\begin{aligned}
(x, y) \in e_{\mathcal{G}} & \operatorname{iff}(\forall B \in \mathcal{G})(y \in B \rightarrow x \in B) \\
& \operatorname{iff}\left(\forall i \in \Gamma, B_{i} \in \mathcal{G}_{\left\langle e_{X_{i}}\right\rangle}\right)\left(y \in \bigcap_{i \in \Gamma} f_{i}^{-1}\left(B_{i}\right)\right. \\
& \left.\rightarrow x \in \bigcap_{i \in \Gamma} f_{i}^{-1}\left(B_{i}\right)\right) \\
& \operatorname{iff}(\forall i \in \Gamma)\left(B_{i} \in \mathcal{G}_{\left\langle e_{X_{i}}\right\rangle}\right)\left(y \in f_{i}^{-1}\left(B_{i}\right) \rightarrow x \in f_{i}^{-1}\left(B_{i}\right)\right) \\
& \operatorname{iff}(\forall i \in \Gamma)\left(\left(f_{i}(x), f_{i}(y)\right) \in e_{\left.\mathcal{G}_{\left\langle e_{X}\right\rangle}\right)}\right. \\
& \operatorname{iff}(x, y) \in e_{\mathcal{G}_{\left\langle e_{X}\right\rangle}}=e_{X}(\text { by Theorem } 2.6(5)) .
\end{aligned}
$$

(4) Let $y \in f_{i}\left(\left\langle e_{X}\right\rangle(A)\right)$ with $x \in\left\langle e_{X}\right\rangle(A)$ and $y=f_{i}(x)$. Then $(x, z) \in e_{X} \& z \in A$ implies $\left(f_{i}(x), f_{i}(z)\right) \in e_{X_{i}} \& f_{i}(z) \in f_{i}(A)$. Then 
$y=f_{i}(x) \in\left\langle e_{X_{i}}\right\rangle\left(f_{i}(A)\right)$. Then $f_{i}\left(\left\langle e_{X}\right\rangle(A)\right) \subset\left\langle e_{X_{i}}\right\rangle\left(f_{i}(A)\right)$. Hence $f_{i}$ is $C$-map for each $i \in \Gamma$. Thus, $\left\langle e_{X}\right\rangle(A) \subset \bigcap_{i \in \Gamma} f_{i}^{-1}\left(\left\langle e_{X_{i}}\right\rangle\left(f_{i}(A)\right)\right)$.

If $\Gamma=\{i\}$, suppose that there exists $A \in P(X)$ with

$$
\left\langle e_{X}\right\rangle(A) \not \supset f_{i}^{-1}\left(\left\langle e_{X_{i}}\right\rangle\left(f_{i}(A)\right)\right) \text {. }
$$

Then there exists $x \in X$ such that

$$
x \in f_{i}^{-1}\left(\left\langle e_{X_{i}}\right\rangle\left(f_{i}(A)\right)\right), \quad x \notin\left\langle e_{X}\right\rangle(A) .
$$

Since $x \in f_{i}^{-1}\left(\left\langle e_{X_{i}}\right\rangle\left(f_{i}(A)\right)\right)$, then $f_{i}(x) \in\left\langle e_{X_{i}}\right\rangle\left(f_{i}(A)\right)$ iff $(\exists z \in$ $X)\left(\left(f_{i}(x), f_{i}(z)\right) \in e_{X_{i}} \& z \in A\right)$ iff $(x, z) \in e_{X} \& z \in A$. Then $x \in$ $\left\langle e_{X}\right\rangle(A)$. It is a contradiction. Hence $\left\langle e_{X}\right\rangle(A) \supset f_{i}^{-1}\left(\left\langle e_{X_{i}}\right\rangle\left(f_{i}(A)\right)\right)$.

(5) Let $y \in f_{i}^{-1}\left(\left[e_{X_{i}}\right](B)\right)$. Then $f_{i}(y) \in\left[e_{X_{i}}\right](B)$. Then $(\forall z \in$ $\left.X_{i}\right)\left(\left(f_{i}(x), z\right) \in e_{X_{i}} \rightarrow z \in B\right)$ implies $(\forall y \in X)\left(\left(f_{i}(x), f_{i}(y)\right) \in e_{X_{i}} \rightarrow\right.$ $\left.f_{i}(y) \in B\right)$ implies $(\forall y \in X)\left((x, y) \in e_{X} \rightarrow y \in f_{i}^{-1}(B)\right)$. Hence $f_{i}^{-1}\left(\left[e_{X_{i}}\right](B)\right) \subset\left[e_{X}\right]\left(f_{i}^{-1}(B)\right)$. So,

$$
\left[e_{X}\right](A) \supset \bigcup_{i \in \Gamma} f_{i}^{-1}\left(\left[e_{X_{i}}\right]\left(\left(f_{i}\left(A^{c}\right)^{c}\right)\right)\right) .
$$

If $\Gamma=\{i\}$, suppose that there exists $A \in P(X)$ with

$$
\left[e_{X}\right](A) \not \subset f_{i}^{-1}\left(\left[e_{X_{i}}\right]\left(\left(f_{i}\left(A^{c}\right)^{c}\right)\right)\right) .
$$

Then there exists $x \in X$ such that

$$
x \notin f_{i}^{-1}\left(\left[e_{X_{i}}\right]\left(\left(f_{i}\left(A^{c}\right)^{c}\right)\right)\right), \quad x \in\left[e_{X}\right](A) .
$$

Since $x \notin f_{i}^{-1}\left(\left[e_{X_{i}}\right]\left(\left(f_{i}\left(A^{c}\right)^{c}\right)\right)\right), f_{i}(x) \in\left(\left[e_{X_{i}}\right]\left(\left(f_{i}\left(A^{c}\right)^{c}\right)\right)\right)^{c}$ iff $f_{i}(x) \in$ $\left(\left\langle e_{X_{i}}\right\rangle\left(f_{i}\left(A^{c}\right)\right)\right)$ iff $\left(\exists y \in X_{i}\right)\left(\left(f_{i}(x), y\right) \in e_{X_{i}} \& y \in f_{i}\left(A^{c}\right)\right)$ iff $(\exists z \in$ $X)\left((x, z) \in e_{X} \& z \in A^{c}\right)$, then $x \notin\left[e_{X}\right](A)$. It is a contradiction. Hence $\left[e_{X}\right](A) \subset f_{i}^{-1}\left(\left[e_{X_{i}}\right]\left(\left(f_{i}\left(A^{c}\right)^{c}\right)\right)\right)$.

(6) Let $A=\bigcup f_{i}^{-1}\left(A_{i}\right) \in \bigcup_{i \in \Gamma} f_{i}^{-1}\left(\mathcal{F}_{\left[e_{X_{i}}\right]}\right)$ with $e_{X_{i}}$-upper set $A_{i}$. Since $x \in \bigcup f_{i}^{-1}\left(A_{i}\right) \&\left(f_{i}(x), f_{i}(y)\right) \in \bigcap_{i \in \Gamma} e_{X_{i}}$ implies $(\exists i \in \Gamma)(x \in$ $f_{i}^{-1}\left(A_{i}\right) \&\left(f_{i}(x), f_{i}(y)\right) \in e_{X_{i}} \rightarrow x \in f_{i}^{-1}\left(A_{i}\right)$, then $A=\bigcup f_{i}^{-1}\left(A_{i}\right)$ is $e_{X}$-upper set;i.e. $e_{X}(A)=A$. Hence $A \in \mathcal{F}_{\left[e_{X}\right]}$. 
If $\Gamma=\{i\}$, let $A \in \mathcal{F}_{\left[e_{X}\right]}$. By (5), Then

$$
A=\left[e_{X}\right](A)=f_{i}^{-1}\left(\left[e_{X_{i}}\right]\left(\left(f_{i}\left(A^{c}\right)^{c}\right)\right)\right) .
$$

Since $\left[e_{X_{i}}\right]\left(\left(f_{i}\left(A^{c}\right)^{c}\right)\right)=\left[e_{X_{i}}\right]\left(\left[e_{X_{i}}\right]\left(\left(f_{i}\left(A^{c}\right)^{c}\right)\right)\right), A \in f_{i}^{-1}\left(\mathcal{F}_{\left[e_{X_{i}}\right]}\right)$.

(7) Let $B=\bigcap f_{i}^{-1}\left(B_{i}\right) \in \bigcap_{i \in \Gamma} f_{i}^{-1}\left(\mathcal{G}_{\left\langle e_{X_{i}}\right\rangle}\right)$ with $e_{X_{i}}^{-1}$-upper set $B_{i}$. Since $y \in \bigcap f_{i}^{-1}\left(B_{i}\right) \&\left(f_{i}(x), f_{i}(y)\right) \in \bigcap_{i \in \Gamma} e_{X_{i}}$ implies $(\forall i \in \Gamma)(x \in$ $\left.f_{i}^{-1}\left(B_{i}\right) \&\left(f_{i}(x), f_{i}(y)\right) \in e_{X_{i}}\right) \rightarrow x \in \bigcap f_{i}^{-1}\left(B_{i}\right)$, then $B \in \mathcal{G}_{\left\langle e_{X}\right\rangle}$.

If $\Gamma=\{i\}$, let $A \in \mathcal{G}_{\left\langle e_{X}\right\rangle}$. By (4), Then

$$
A=\left\langle e_{X}\right\rangle(A)=f_{i}^{-1}\left(\left\langle e_{X_{i}}\right\rangle\left(f_{i}(A)\right)\right) .
$$

Since $\left\langle e_{X_{i}}\right\rangle\left(f_{i}(A)\right)=\left\langle e_{X_{i}}\right\rangle\left(\left\langle e_{X_{i}}\right\rangle\left(f_{i}(A)\right)\right), A \in f_{i}^{-1}\left(\mathcal{G}_{\left\langle e_{X_{i}}\right\rangle}\right)$.

EXAmple 3.4. Let $X=\{a, b, c, d\}, Y=\{x, y, z\}$ and $Z=\{u, v, w\}$ be sets. Let $e_{Y}$ and $e_{z}$ be preodered set as follows

$$
\begin{gathered}
e_{Y}=\{(x, x),(y, y),(z, z),(x, z),(z, y)(x, y)\} \\
e_{Z}=\{(u, u),(v, v),(w, w),(u, v),(v, w)(u, w)\}
\end{gathered}
$$

Define $f_{1}: X \rightarrow Y$ and $f_{2}: X \rightarrow Z$ as

$$
\begin{aligned}
& f_{1}(a)=x, f_{1}(b)=f_{1}(c)=y, f_{1}(d)=z, \\
& f_{2}(a)=f_{2}(b)=u, f_{1}(c)=v, f_{2}(d)=w .
\end{aligned}
$$

We obtain

$$
\begin{gathered}
e_{X}=\{(a, a),(a, b),(a, c),(a, d),(b, b),(b, c),(c, c), \quad(c, d),(d, d)\}, \\
{\left[e_{Y}\right](A)= \begin{cases}\{y\} & \text { if }\{y\} \subset A,\{y, z\} \not \subset A, \\
\{y, z\} & \text { if } A=\{y, z\} \\
Y & \text { if } A=Y, \\
\emptyset & \text { otherwise, }\end{cases} }
\end{gathered}
$$




$$
\begin{aligned}
& {\left[e_{Z}\right](B)= \begin{cases}\{w\} & \text { if }\{w\} \subset A,\{v, w\} \not \subset A, \\
\{v, w\} & \text { if } A=\{v, w\} \\
Z & \text { if } A=Y, \\
\emptyset & \text { otherwise, }\end{cases} } \\
& {\left[e_{X}\right](A)= \begin{cases}\{d\} & \text { if }\{d\} \subset A,\{c\} \not \subset A, \\
\{c\} & \text { if }\{c\} \subset A,\{d\} \not \subset A, \\
\{b, c\} & \text { if }\{b, c\} \subset A,\{d\} \not \subset A, \\
\{c, d\} & \text { if }\{c, d\} \subset A,\{b\} \not \subset A, \\
\{b, c, d\} & \text { if } A=\{b, c, d\}, \\
X & \text { if } A=X, \\
\emptyset & \text { otherwise, }\end{cases} }
\end{aligned}
$$

Then $\left[e_{X}\right](A) \supset \bigcup_{i \in \Gamma} f_{i}^{-1}\left(\left[e_{X_{i}}\right]\left(\left(f_{i}\left(A^{c}\right)^{c}\right)\right)\right)$. In general,

$$
\{c\}=\left[e_{X}\right](\{c\}) \neq \bigcup_{i \in \Gamma} f_{i}^{-1}\left(\left[e_{X_{i}}\right]\left(\left(f_{i}\left(\{c\}^{c}\right)^{c}\right)\right)\right)=\emptyset
$$

Moreover,

$$
\begin{aligned}
& \mathcal{F}_{\left[e_{Y}\right]}=\{\emptyset,\{y\},\{y, z\}, Y\}, \quad \mathcal{F}_{\left[e_{Z}\right]}=\{\emptyset,\{w\},\{v, w\}, Z\}, \\
& \mathcal{F}_{\left[e_{X}\right]}=\{\emptyset,\{c\},\{d\},\{b, c\},\{c, d\},\{b, c, d\}, X\} .
\end{aligned}
$$

$$
\begin{aligned}
& \left\langle e_{Y}\right\rangle(A)= \begin{cases}Y & \text { if }\{y\} \subset A, \\
\{x, z\} & \text { if }\{z\} \subset A,\{y\} \not \subset A, \\
\{x\} & \text { if }\{x\} \subset A,\{z\} \not \subset A, \\
\emptyset & \text { if } A=\emptyset,\end{cases} \\
& \left\langle e_{Z}\right\rangle(B)= \begin{cases}Z & \text { if }\{w\} \subset A, \\
\{u, v\} & \text { if }\{v\} \subset A,\{u\} \not \subset A, \\
\{u\} & \text { if }\{u\} \subset A,\{v\} \not \subset A, \\
\emptyset & \text { if } A=\emptyset,\end{cases}
\end{aligned}
$$




$$
\left\langle e_{X}\right\rangle(A)= \begin{cases}\{a\} & \text { if }\{a\} \subset A,\{b\} \not \subset A,\{d\} \not \subset A \\ \{a, b\} & \text { if }\{b\} \subset A,\{d\} \not \subset A, \\ \{a, d\} & \text { if }\{d\} \subset A,\{b\} \not \subset A, \\ \{a, b, c\} & \text { if }\{c\} \subset A,\{d\} \not \subset A, \\ \{a, b, d\} & \text { if } A=\{a, b, d\}, \\ \emptyset & \text { if } A=\emptyset, \\ X & \text { otherwise, }\end{cases}
$$

Then $\left\langle e_{X}\right\rangle(A) \subset \bigcap_{i \in \Gamma} f_{i}^{-1}\left(\left\langle e_{X_{i}}\right\rangle\left(\left(f_{i}(A)\right)\right)\right)$. In general,

$$
\begin{aligned}
\{a, b, d\} & =\left\langle e_{X}\right\rangle(\{a, b, d\}) \\
& \neq \bigcap_{i \in \Gamma} f_{i}^{-1}\left(\left\langle e_{X_{i}}\right\rangle\left(f_{i}(\{a, b, d\})\right)\right)=X .
\end{aligned}
$$

Moreover,

$$
\begin{aligned}
& \mathcal{G}_{\left\langle e_{Y}\right\rangle}=\{\emptyset,\{x\},\{x, z\}, Y\}, \mathcal{G}_{\left\langle e_{Y}\right\rangle}=\{\emptyset,\{u\},\{u, v\}, Z\} \\
& \mathcal{G}_{\left\langle e_{X}\right\rangle}=\{\emptyset,\{a\},\{a, d\},\{a, b\},\{a, b, d\},\{a, b, c\}, X\} .
\end{aligned}
$$

Using Theorem 3.3, we can define subspaces and products in the obvious way.

Definition 3.4. Let $\left(X, e_{X}\right)$ be a preordered space and $A$ a subset of $X$. The pair $\left(A, e_{A}\right)$ is said to be a subspace of $\left(X, e_{X}\right)$ if $e_{A}$ is the coarsest preorder on $A$ which the inclusion function $i: A \rightarrow X$ is an ordered preserving map.

Definition 3.5. Let $\left\{\left(X_{i}, e_{X_{i}}\right) \mid i \in \Gamma\right\}$ be a family of preordered spaces. Let $X=\prod_{i \in \Gamma} X_{i}$ be a product set. The coarsest preorder $e_{X}=\otimes e_{X_{i}}$ on $X$ with respect to $\left(X, \pi_{i},\left(X_{i}, e_{X_{i}}\right)\right)$ where $\pi_{i}: X \rightarrow X_{i}$ is projection map is called the product preorder of $\left\{e_{X_{i}} \mid i \in \Gamma\right\}$.

\section{References}

[1] R. Bělohlávek, Lattices of fixed points of Galois connections, Math. Logic Quart. 47 (2001), 111-116.

[2] G. Georgescu, A. Popescue, Non-dual fuzzy connections, Arch. Math. Logic. 43 (2004), 1009-1039. 
[3] J. Järvinen, M. Kondo, J. Kortelainen, Logics from Galois connections, Internat. J. Approx. Reason. 49 (2008), 595-606.

[4] Y.C. Kim, Y.S. Kim, Relations and upper sets on partially ordered sets, Int. Math. Forum 6 (19) (2011), 899-908.

[5] Y.C. Kim, Y.S. Kim, Various connections on interior sets, Int. Math. Forum 6 (19) (2011), 909-920.

[6] Y.C. Kim, Y.S. Kim, Rough approximations on preordered sets, accepted to, Int. J. Fuzzy. Int. Sys.

[7] Z. Pawlak, Rough sets, Int. J. Comput. Inf. Sci. 11 (1982), 341-356.

[8] G. Qi, W. Liu, Rough operations on Boolean algebras, Information Sciences 173 (2005), 49-63.

Department of Mathematics, Natural Science

Gangneung-Wonju National University

Gangneung, Gangwondo, 210-702, Korea

E-mail: yck@gwnu.ac.kr

Department of Mathematics, Natural Science

Gangneung-Wonju National University

Gangneung, Gangwondo, 210-702, Korea

E-mail: jmko@gwnu.ac.kr 